population is required. To examine the ability of GMS to map genetic traits on a genome-wide scale, we performed selections for regions of IBD between multiple parent/segregant pairs of Saccharomyces cerevisiae to define candidate regions for loci of single and multigenic traits. DNA recovered from GMS of reciprocal parent/segregant pairs was amplified, differentially labelled with fluorescent probes and hybridised to microarrays containing all known yeast open reading frames. The degree of selection for regions of IBD was sufficient to allow determination of the parental genotype for approximately $85 \%$ of all open reading frames within the genome of each segregant based on a threshold ratio of 1.25 . Our study results demonstrated that GMS was able to identify correct regions of the genome for all single gene traits examined. We were also able to identify two candidate regions for a multigenic trait predicted to result from the inheritance of two alleles.

Hegde, Priti

\section{Identification of genes involved in colon cancer metastasis using cDNA microarrays}

Priti Hegde ${ }^{1}$, Julie Earle-Hughes ${ }^{1}$, Kristie Abemathy ${ }^{1}$, Cheryl Gay ${ }^{1}$, Sonia Dharap, Alexander I. Saeed ${ }^{1}$, Vasily Sharov, Norman H. Lee $^{1}$, Timothy Yeatman ${ }^{2} \&$ John Q uackenbush ${ }^{1}$

${ }^{1}$ The Institute for Genomic Research, Rockville, M aryland 20850, USA

${ }^{2}$ The H. Lee M offitt Cancer Center, Tampa, Florida, USA

Colon cancer is the second most common cause of cancer-related deaths in the United States. Despite significant advances in therapy, poor prognosis of colon cancer metastasis results in high mortality. Metastasis occurs due to defects in multiple genes and consists of a series of complex sequential events that define the ultimate stage of tumour progression. Unlike tumourgenesis, which requires presence of mutated genes, metastasis is mostly a result of altered expression of genes. cDNA microarrays provide an ideal tool for the high-throughput analysis of gene function on a genome-wide scale. We assembled a collection of cDNA clones representing more than 40,000 distinct genes, developed laboratory hardware and protocols, and created databases and data analysis tools necessary for analysing differential expression. We used High-density cDNA microarrays containing more than 19,200 PCR-amplified clones to study differential expression patterns between less metastatic (KM12C) and highly metastatic (KM12L4A, KM12SM) cellular phenotypes in human colon carcinoma cell lines. Our statistical analysis of the expression ratios calculated from the measured fluorescent intensities suggests genes of prognostic or diagnostic value. Identification of these genes would assist in providing a more complete understanding of gene function and regulation with respect to cancer metastasis.

Heiskanen, Mervi A.

\section{Direct identification of amplified genes in human cancers by genomic hybridization to CDNA microarrays}

\author{
M. Heiskanen ${ }^{1}$, M. Bittner ${ }^{1}$, Y. Chen, K. Adler ${ }^{2}$, J.M. Trent ${ }^{1}$ \\ \& P.S. M eltzer ${ }^{1}$
}

${ }^{1}$ National Human Genome Research Institute, Bethesda, M aryland 20892, USA ${ }^{2}$ NEN Life Science Products, Inc., Boston, M assachusetts 02118, USA

Gene amplification is one of the major mechanisms of oncogene activation in tumorigenesis. Currently, however, direct identification of amplified genes in cancer remains difficult. The most important technique allowing genome-wide screening of gene copy-number changes is comparative genomic hybridization
(CGH). CGH is based on the hybridization of equal amounts of normal and tumour DNA in situ on normal metaphase chromosomes. DNAs are labelled with different fluorochromes and differences in the intensity ratios indicate copy-number differences in the amount of sample and reference DNA (ref. 1). CGH only allows the detection of copy-number changes on a megabase scale, however, and the genes resident in amplicons must be identified using other techniques. We have developed a technique based on the hybridization of total genomic DNA on cDNA microarrays. These microarrays contain thousands of known genes and unknown ESTs. Compared with the previously published genomic microarray techniques where genomic DNA is hybridized on genomic clones (CGH microarrays), hybridization on cDNAs offers some advantages. The most important is the possibility to directly identify amplified genes, not just amplified genomic regions. Due to the complexity of genomic DNA and the small size of the arrayed probe $(0.5-2 \mathrm{~kb})$, however, the hybridization signals are weak. To facilitate signal detection, we have used a tyramide-based technique that allows amplification of a fluorescent signal up to 1,000-fold. We hybridized seven different cancer cell lines on cDNA microarrays. We detected the amplification of several genes, including C-myc, N-myc, SAS, MDM2, ERBB2 and OS4. The sensitivity of this assay was studied by diluting tumour DNA with normal genomic DNA, and the results suggest that amplifications of fivefold and higher can be detected. An additional advantage of this technique is the potential to measure copy number and gene expression for the same set of genes by hybridizing either DNA or cDNA on identical arrays. This will eventually allow correlation of gene amplification with gene expression on a genome-wide scale. We think that this technique is very likely to become an important tool for screening and discovery of genes important in tumorigenesis.

1. Kallioniemi, A. et al. Science 258, 818-821 (1992)

Hodgson, J. Graeme

\section{High-resolution assessment of DNA loss in RIP-Tag mouse pancreatic carcinomas using comparative genomic hybridization to microarrays}

\author{
Graeme Hodgson'1, Jeff Hager', Colin Collins ${ }^{1}$, Donna Albertson'1, \\ Daniel Pinkel ${ }^{1}$, Doug Hanahan' ${ }^{2} \&$ Joe W. Gray ${ }^{1}$ \\ ${ }^{1}$ UCSF Cancer Center, San Francisco, California, USA \\ ${ }^{2}$ Departments of Biochemistry and Biophysics, UCSF, \\ San Francisco, California, USA
}

Transgenic expression of the SV40 large T-antigen under control of the insulin gene regulatory region in the RIP-Tag mouse model results in development of islet -cell carcinomas. Tumorigenesis involves a distinct progression as the islets move from normal quiescence to a hyperproliferative state, followed by angiogenic induction and finally to reduced apoptosis, resulting in solid tumour formation. $\mathrm{LOH}$ and CGH analyses have identified putative tumour-suppressor genes, as recurrent loss of DNA on chromosomes 16 (LOH16) and 9 (LOH9) have been observed corresponding to the angiogenic and reduced apoptosis switches in tumour development, respectively. Although partial loss of chromosomal DNA in some tumours and tumour-derived cell lines (TCL) has narrowed the regions of interest, the regions remain large ( $\sim 15 \mathrm{Mb}$ on chromosome 9 and $\sim 12 \mathrm{Mb}$ on chromosome 16) and no known tumour-suppressor genes are known to map to these loci. To further delineate each region and to facilitate gene discovery, we are using an array-CGH (CGHa) approach using BAC clones as array elements that map to LOH9 or LOH16. Thus far, 12 BACs have been isolated from the LOH9 region based on known marker and gene sequence information available in public databases and FISH mapped to confirm chromosomal location. The clones are then arrayed onto silynated glass slides using using a high-throughput, custom-made arraying device developed at UCSF. Additional BAC and P1 clones known to map elsewhere in the mouse genome serve as controls in the CGHa analyses. Tumour 